\title{
DILEMAS ÉTICOS VIVENCIADOS PELA EQUIPE DE SAÚDE NO CUIDADO DA PESSOA EM TRATAMENTO ONCOLÓGICO
}

\author{
ETHICAL DILEMMAS EXPERIENCED BY \\ THE HEALTH TEAM IN THE CARE OF \\ THE PERSON IN CANCER TREATMENT
}

\section{DILEMAS ÉTICOS EXPERIMENTADOS POR EL EQUIPO DE SALUD EN EL CUIDADO DE LA PERSONA EN TRATAMIENTO ONCOLÓGICO}

\author{
Maryana Carneiro de Queiroz Ferreira ${ }^{1}$ \\ Marluce Alves Nunes Oliveira ${ }^{2}$ \\ Thamara Arianny Ventin Amorim Oliveira de Assis ${ }^{3}$ \\ Elaine Guedes Fontoura ${ }^{4}$ \\ Manuela Bezerra Pina Oliveira \\ Keccya dos Santos Nunes Gonçalves \\ Joselice Almeida Gois
}

Como citar este artigo: Ferreira MCQ, Oliveira MAN, Assis TAVAO, Fontoura EG, Oliveira MBP, Gonçalves KSN, et al. Dilemas éticos vivenciados pela equipe de saúde no cuidado da pessoa em tratamento oncológico. Rev baiana enferm. 2021;35:e43346.

\begin{abstract}
Objetivo: conhecer os dilemas éticos vivenciados na prática da equipe de saúde no cuidado da pessoa em tratamento oncológico. Método: estudo exploratório e descritivo de abordagem qualitativa realizado em uma unidade de assistência de alta complexidade em oncologia em um município do interior da Bahia, Brasil. Foi realizada entrevista semiestruturada, que contou com a participação de onze profissionais da área da saúde. A análise dos dados ocorreu utilizando-se a técnica de Análise de Conteúdo proposta por Bardin. Resultados: o estudo apontou que os profissionais de saúde que atuam em cuidados oncológicos vivenciam dilemas éticos na prática, frente às intervenções nos cuidados paliativos, tais como não revelar o diagnóstico à pessoa com câncer e dúvida quanto às manobras para reanimar ou não. Conclusão: os profissionais de saúde vivenciam dilemas éticos cotidianamente e, em sua maioria, possuem conhecimento sobre eles, embora tenha sido encontrada ambiguidade entre os termos conflito ético e dilema ético.
\end{abstract}

Descritores: Ética. Oncologia. Equipe de Assistência ao Paciente.

\footnotetext{
Estudante de Enfermagem. Universidade Estadual de Feira de Santana. Feira de Santana, Bahia, Brasil. http://orcid.org/0000-0002-57।I-4485.

Enfermeira. Doutora em Enfermagem. Docente da Universidade Estadual de Feira de Santana. Feira de Santana, Bahia, Brasil. milicialves@yahoo.com.br. http://orcid.org/0000-0002-9109-0106.

3 Enfermeira. Universidade Estadual de Feira de Santana. Feira de Santana, Bahia, Brasil. http://orcid.org/0000-00023356-8044.

4 Enfermeira. Doutora em Enfermagem. Docente da Universidade Estadual de Feira de Santana. Feira de Santana, Bahia, Brasil. http://orcid.org/0000-00023434-22I5.

5 Enfermeira. Coordenadora da Comissão de Controle de Infecção Hospitalar do Hospital Dom Pedro de Alcântara. Feira de Santana, Bahia, Brasil. http://orcid. org/0000-0002-8407-0553.

Enfermeira. Universidade Estadual de Feira de Santana. Feira de Santana, Bahia, Brasil. http://orcid.org/0000-0002-3|22-93।3.

Enfermeira. Mestre em Enfermagem. Docente da Universidade Estadual de Feira de Santana. Feira de Santana, Bahia, Brasil. http://orcid.org/0000-000 I-8870-3509.
} 
Objective: to know the ethical dilemmas experienced in the practice of the health team in the care of the person in cancer treatment. Method: exploratory and descriptive study of qualitative approach conducted in a unit of high complexity assistance in oncology in a municipality in the countryside of Babia, Brazil. A semi-structured interview was conducted, with the participation of eleven health professionals. Data analysis occurred using the Content Analysis technique proposed by Bardin. Results: the study pointed out that health professionals working in cancer care experience ethical dilemmas in practice, before interventions in palliative care, such as not revealing the diagnosis to the person with cancer and doubt as to the maneuvers to revive or not. Conclusion: health professionals daily experience ethical dilemmas and most have knowledge about them, although ambiguity has been found between the terms ethical conflict and ethical dilemma.

Descriptors: Ethics. Oncology. Patient Assistance Team.

Objetivo: conocer los dilemas éticos experimentados en la práctica del equipo de salud en el cuidado de la persona en tratamiento oncológico. Método: estudio exploratorio y descriptivo de abordaje cualitativo realizado en una unidad de asistencia de alta complejidad en oncología en un municipio del interior de Babía, Brasil. Se realizó una entrevista semiestructurada, con la participación de once profesionales de la salud. El análisis de los datos se produjo mediante la técnica de Análisis de Contenido propuesta por Bardin. Resultados: el estudio señaló que los profesionales de la salud que trabajan en la atención oncológica experimentan dilemas éticos en la práctica, frente a las intervenciones en cuidados paliativos, como no revelar el diagnóstico a la persona con cáncer y tener dudas sobre las maniobras para revivir o no. Conclusión: los profesionales de la salud experimentan dilemas éticos a diario $y$, en su mayor parte, tienen conocimiento sobre ellos, aunque se ha encontrado ambigüedad entre los términos conflicto ético y dilema ético.

Descriptores: Ética. Oncología. Equipo de Asistencia al Paciente.

\section{Introdução}

A ética, ao longo do tempo, vem sendo aprimorada de acordo com a evolução da sociedade. Considera-se que tal evolução promoveu a liberdade de ação do profissional que cuida, bem como o respeito à autonomia da pessoa adoecida e de seus familiares. Dessa forma, o cuidado da equipe de saúde incorpora, no dia a dia, perspectivas pessoais, teóricas e práticas, éticas, morais e legais e promove reflexão acerca da atenção e do respeito à dignidade da pessoa doente.

Os dilemas éticos emergem tanto na vida pessoal quanto profissional das pessoas. Esses podem ser de ordem administrativa ou relacionados aos problemas éticos e morais. Então, por ser a ética necessária na prática profissional, ao abordar-se pessoas adoecidas, em especial pessoas com câncer, é necessária uma reflexão constante a respeito dos problemas éticos, a fim de que sejam traçadas estratégias de enfrentamento ${ }^{(1)}$.

O câncer é mundialmente considerado um problema de saúde pública de grande relevância epidemiológica, no que tange à incidência e à morbimortalidade ${ }^{(1)}$. As pessoas com o diagnóstico de câncer experimentam alterações tanto físicas quanto psicológicas que podem ter início na revelação do diagnóstico, no tratamento e na pós-recuperação. Nesse momento, a pessoa vivencia efeitos colaterais dos medicamentos, comorbidades e deterioração da funcionalidade do organismo, além de experienciar ansiedade, medo, tristeza e outros sentimentos em todos os estágios da doença. Tais sentimentos são também experimentados pelos familiares, que enfrentam uma carga mental pesada junto com seu ente querido, porquanto essa doença requer uma equipe multidisciplinar em cuidados oncológicos que assista a todos os envolvidos e promova qualidade de vida ${ }^{(2)}$.

A equipe multidisciplinar, ao prestar os cuidados oncológicos, pode vivenciar dilemas éticos difíceis de serem resolvidos, que requerem conhecimento e agir ético-profissional, além de desvelo na relação interpessoal, para que os valores das pessoas em tratamento oncológico, dos familiares e dos profissionais sejam respeitados e o cuidado prestado seja ético e digno( ${ }^{(3)}$. 
Este estudo foi motivado pela vivência na prática como integrantes da equipe de saúde que cuida de pessoas em tratamento oncológico e por perceber-se que os dilemas éticos ocorrem e muitas vezes não são identificados. Então, surgiu a questão de investigação: Como a equipe de saúde vivencia os dilemas éticos no cuidado da pessoa em tratamento oncológico?

Considera-se este estudo de grande relevância, por possibilitar conhecer os dilemas éticos vivenciados pela equipe de saúde no cuidado da pessoa em tratamento oncológico no contexto hospitalar, bem como promover reflexão, para que, diante deles, as pessoas que compõem a equipe tenham capacidade de tomar decisões.

Este estudo tem como objetivo conhecer os dilemas éticos vivenciados na prática da equipe de saúde no cuidado da pessoa em tratamento oncológico.

\section{Método}

Consiste em um estudo exploratório e descritivo de abordagem qualitativa, que teve como objeto de estudo os "dilemas éticos vivenciados no cuidado da pessoa em tratamento oncológico”. É parte do Projeto de Pesquisa intitulado "Conflitos e Dilemas Éticos Vividos no Cuidado da Equipe de Saúde no Contexto Hospitalar", com Resolução n 016/2018 do Conselho Superior de Ensino, Pesquisa e Extensão (CONSEPE).

O estudo foi realizado em uma Unidade de Assistência de Alta Complexidade em Oncologia, de referência, localizada numa cidade de grande porte no interior do estado da Bahia, Brasil. A unidade de oncologia foi inaugurada em setembro de 2009 e conta com um ambulatório onde são realizadas as consultas com as equipes clínicas, cirúrgicas e de radioterapia, além dos tratamentos ambulatoriais de quimioterapia, imunoterapia, hormonioterapia e radioterapia.

É constituída por uma equipe multiprofissional composta por enfermeiras, médicos, nutricionistas, psicólogos, farmacêuticos, fonoaudiólogos, assistentes sociais, fisioterapeutas, recepcionistas, técnicos de enfermagem de radiologia e físico médico.
Os participantes investigados foram 11 profissionais da área da saúde: oito enfermeiras, um médico, uma nutricionista e uma fisioterapeuta. Adotou-se como critério de inclusão: desenvolver atividade assistencial por mais de um ano. O critério de exclusão foi encontrar-se afastado por férias ou licença.

A coleta de dados foi realizada por uma das autoras e membro do Núcleo Interdisciplinar de Pesquisas e Estudos em Saúde (NIPES), no período de junho a outubro de 2018.

No primeiro momento, entrou-se em contato com a coordenação de Enfermagem, para esclarecer sobre a temática da pesquisa, objetivo e justificativa do estudo. Em seguida, os profissionais foram convidados para participar da pesquisa. Àqueles que aceitaram, foi entregue o Termo de Consentimento Livre e Esclarecido (TCLE), com a orientação de que devia ser lido e assinado em duas vias.

A coleta de dados foi realizada por meio de entrevista semiestruturada, audiogravada, com média de 3,23 minutos para as questões norteadoras e de 1,0 minuto para a caracterização, de acordo com a disponibilidade dos participantes, em dia e local determinado por eles. O maior número de enfermeiras a serem entrevistadas decorreu do fato de considerar-se que realizavam o cuidado diuturnamente e terem a possibilidade de vivenciar dilemas éticos. Vale ressaltar que a interrupção da coleta de dados, bem como a definição do número de participantes, ocorreu com a saturação teórica, na décima primeira entrevista.

O instrumento de coleta de dados foi dividido em duas partes: a primeira, contemplou a caracterização dos participantes - idade, sexo, tempo de formado, tempo de atuação em unidade oncológica, carga horária semanal, outros vínculos empregatícios, outros setores de atuação, titulação e pós-graduação; a segunda compôs-se de questões norteadoras: O que você entende por dilemas éticos? Fale-me de dilemas éticos vivenciados por você na prática do cuidado de pessoas em tratamento oncológico.

Para organização e posterior análise dos dados, utilizou-se a técnica de Análise de 
Conteúdo proposta por Bardin ${ }^{(4)}$, que se divide em três etapas: a primeira, pré-análise, em que foram organizados os materiais que seriam úteis para a pesquisa, com uma análise sistematizada, a fim de conduzir um esquema preciso do desenvolvimento das operações sucessivas; a etapa seguinte, exploração do material, consistiu numa descrição analítica dos dados; na última etapa, realizaram-se inferências e interpretações baseadas no objetivo proposto ${ }^{(4)}$.

Para garantir o anonimato, os participantes foram identificados pela letra inicial da sua profissão seguida do número correspondente à ordem da entrevista: E1, E2... (Enfermeira); N1 (Nutricionista); F1 (Fisioterapeuta); e M1 (Médico).

O projeto foi submetido ao Comitê de Ética em Pesquisa (CEP) da Universidade Estadual de Feira de Santana (UEFS) e aprovado pelo Certificado de Apresentação de Apreciação Ética (CAAE) $\mathrm{n}^{\circ}$ 2865214.9.0000.0053.

\section{Resultados e Discussão}

Foram entrevistados 11 profissionais da área de saúde que atuavam nas unidades de tratamento oncológico. Oito eram do sexo feminino e 3, do masculino, com faixa etária entre 27 e 36 anos. Quanto à formação, 8 eram enfermeiras, 1 nutricionista, 1 fisioterapeuta e 1 médico. Tempo de formação de 1 ano e 6 meses a 10 anos, que correspondeu ao tempo de atuação em unidades oncológicas da instituição pesquisada.

A leitura dos relatos tornou possível identificar três categorias: Como a equipe de saúde entende o dilema ético, Dilemas éticos diante da pessoa em cuidados paliativos e Ética.

\section{Como a equipe de saúde entende o dilema ético}

Nesta categoria, os profissionais da equipe expressaram que entendiam os dilemas éticos como uma necessidade de tomar uma decisão que ia de encontro aos seus valores e se tornava uma barreira para o seu fazer e agir éticos.

Nas falas de E1 e E2, percebe-se que, diante da situação em que existem duas opções de escolha, o profissional tem que tomar uma decisão. Então, a partir do momento que a decisão afeta os seus princípios éticos e morais, surgem as dúvidas e estabelece-se o dilema ético. Situação na qual você, enquanto profissional, tem que tomar
uma atitude [...] [que] na maioria das vezes, pode afetar de
alguma forma a sua, sua moral, seus, seus costumes. (E1).

Você ter dois caminhos pra seguir e você é..., ter que optar por um dos caminhos, sendo que você não, não consegue identificar o que é que vai ser melbor para aquele momento. (E2).

Para E3, E4 e E6, o profissional de saúde vivencia o dilema ético no momento em que, mesmo sabendo que vai de encontro à ética profissional, deve decidir frente a uma situação em que uma barreira o impede de tomar a decisão mais adequada.

\footnotetext{
Algumas situações em que, às vezes, a gente se depara no decorrer da profissão, referente a condutas éticas que, na maioria das vezes, você não consegue saber, na verdade, como se sair diante de algumas situações. (E3).

Você saber o que é algo, o correto a fazer e ter uma barreira que te impeça a fazer, porque você fica no meio de uma determinada situação, né? De um lado e de outro, mas nunca fugindo do que é o certo, mas você não pode modificar aquilo. (E4).

Bom... que pergunta difícil! Bom... Pra mim, eu não sei se vou falar certo, não, mas, pra mim, dilemas a gente até vivencia muito aqui. A gente tem, na verdade..., quando a gente estuda, né? A gente tem um dilema a seguir que é ético, e normalmente a gente acaba tendo que não seguir aquilo que, pra gente, seria ético. Então, é um dilema pra gente. É uma coisa, não que a gente faz errado, mas são coisas fora do que a gente pode fazer em relação à ética. (E6).
}

Então, para o participante E3, o dilema apresenta-se no exercício da profissão, quando se depara com situações que não sabe de fato como decidir. Para E4, o dilema é definido como uma barreia diante de determinada situação, que pode resultar em alterações das condutas, de acordo com o caso. Segundo E6, o dilema ético vai ser caracterizado como uma situação em que se faz algo, contrariando os valores éticos.

Em vista disso, associa-se aos resultados expostos a situação em que o profissional de saúde defronta-se com o dilema ético, ao ter de optar por uma das alternativas possíveis em determinado caso, mas ambas apresentam vantagens e desvantagens éticas. Desse modo, a decisão pode ir de encontro aos princípios éticos e morais que orientam sua conduta ${ }^{(5)}$. 
[Suspirou!] Boa pergunta! [risos] Rapaz, é tudo que envolve tanto a ética dentro da nossa profissão, dentro das condutas éticas como enfermeiro. É... como os dilemas que os pacientes mesmos nos trazem, dentro daquilo que eles vivem, além da própria doença... (E8).

E8 relata que o dilema ético está relacionado com as experiências profissionais e os problemas da pessoa em tratamento oncológico. Nessa perspectiva, o dilema ético nem sempre se refere aos aspectos éticos diretamente, mas pode estar vinculado à esfera pessoal, incorporado à vida individual de cada profissional e das pessoas que cuidam ${ }^{(6)}$.

O dilema ético, na visão de F1, está relacionado com o conflito para prosseguir diante de uma situação, quando não se sabe distinguir o conflito ético do dilema ético. Esse impasse evidencia a possibilidade de equívocos quanto ao entendimento desses termos.

Eu entendo que dilema seja conflito, né? Um conflito que você viva em determinada situação e, e... Um dilema ético é, por exemplo, você tem que seguir a ética em tal situação, a ética da sua profissão, por exemplo, e você tem um conflito em seguir aquilo ali, então, como se fosse uma dificuldade em realizar aquele procedimento de forma ética... (F1).

Dilemas éticos dizem respeito às situações de difícil decisão, em que não há uma solução linear, mas um confronto de valores. A solução do dilema requer o envolvimento individual responsável e reflexivo, com questionamentos no momento da decisão ${ }^{(7)}$, uma vez que é preciso avaliar todas as alternativas, a fim de deliberar com mais clareza ${ }^{(5)}$. Já o conflito ético é caracterizado como uma divergência e diversidade de opiniões que abrange pessoas com percepções diferentes, causando discussões, sendo necessário um consenso entre os distintos pontos de vista. Nesse sentido, por serem comumente utilizados para referir um mesmo domínio de reflexão, fica clara a ambiguidade desses termos para definir e diferenciar o entendimento de dilema e conflito ético ${ }^{(8)}$.

Neste estudo, os resultados revelaram que os profissionais de saúde, em sua maioria, compreendiam o significado de dilemas éticos. Um deles, entretanto, equivocou-se ao expor o conceito de dilema e de conflito ético. Logo, a compreensão dos dilemas éticos pode ser importante aspecto na prática profissional de pessoas éticas, uma vez que possibilita a tomada de decisão consciente.

\section{Dilemas éticos diante da pessoa em cuidados paliativos}

Nesta categoria, os achados do estudo demonstraram que dilemas éticos estavam associados aos cuidados paliativos da pessoa com câncer, visto que, em alguns casos, ela necessita desse tipo de cuidados, que nem sempre são proporcionados. Outra situação observada diz respeito a não reconhecer os limites do que pode fazer por aquela pessoa e querer salvar sua vida, ainda que nem sempre seja possível.

Para E1 e E2, os dilemas éticos que vivenciavam estavam relacionados a determinar ou não cuidados paliativos para uma pessoa. Associavam-se a isso os dilemas causados na equipe de saúde, motivados pelo desconhecimento do conceito, pois geravam dificuldades e questionamentos para prestar o cuidado de forma ética e qualificada.

Os dilemas éticos com os quais eu vivenciei durante o tempo da enfermaria, foram os dilemas nos quais os pacientes tinham prognóstico de cuidado paliativo. Esse prognóstico de cuidado paliativo não tava no prontuário, assinado pelo médico, e você vivenciar o conflito ético em, no momento que esse paciente parava, se você reanimava ou não. (E1).

O principal dilema boje aqui na clínica médica [...] o paciente não ser definido como cuidado paliativo, porém você enxergar que esse paciente era pra ser um paciente de cuidado paliativo e o médico oncologista tem essa dificuldade em fazer essa definição e escrever no prontuário, porque não basta ele definir [...] tem que escrever no prontuário que é um paciente de cuidados paliativos, para que a equipe fique ciente e possa dar, dar prosseguimento aos cuidados e ao tratamento desse paciente. (E2).

Cuidado paliativo é considerado um cuidado integral, que visa à qualidade de vida da pessoa com uma doença ameaçadora, incluindo aspectos físicos, psicológicos, sociais e espirituais. Além disso, visa promover a prevenção e o alívio do sofrimento tanto para as pessoas acometidas pela doença quanto para seus familiares ${ }^{(9)}$.

Nesse contexto, em presença de um diagnóstico clínico, é dever do médico definir o diagnóstico e o prognóstico, registrando-os no prontuário e mantendo uma comunicação com a equipe, a fim de que a assistência interdisciplinar 
seja realizada em consonância e com o melhor tratamento para tal, de modo a atingir a evolução esperada. Além disso, esse encaminhamento respalda situações profissionais que necessitem ser $\operatorname{avaliadas}^{(1,10)}$.

\begin{abstract}
A única coisa que se encaixa é o paciente paliativo, você querer investir mais. O mais complicado é isso [...] porque a família quer que você faça mais e você sabe que, mesmo que você faça mais, não vai ter o resultado esperado e, de certa forma, você também não pode passar por cima disso... (F1).
\end{abstract}

F1 revela a vivência de dilemas, quando se encontra diante de uma pessoa em cuidados paliativos e, como profissional, não saber até onde pode investir naquela pessoa. Há ainda o aspecto de a família querer mais investimento e o profissional encontrar-se diante do dilema entre fazer ou não fazer mais pela pessoa.

Em concordância com a literatura, diante de uma pessoa com uma doença avançada, em cuidados paliativos, os profissionais de saúde podem encontrar-se diante de um dilema ético, ao ter que avaliar risco e benefício quanto aos tratamentos e cuidados a serem realizados, tendo como foco principal a maximização dos benefícios e que os cuidados garantam eficácia e eficiência ${ }^{(11)}$.

Para M1, um dos dilemas éticos vivenciados pelos profissionais que cuidam da pessoa com problemas oncológicos pode estar relacionado à questão de cuidados paliativos. O depoente esclarece que não se trata de salvar vida ou prolongá-la, mas, sim, de dar qualidade de vida à pessoa que vivencia uma doença sem possibilidade de cura, bem como apoiar a família diante dos sofrimentos enfrentados.

Então, um dos dilemas éticos que a gente trabalba é exatamente esse. É a questão de paliação. A gente lidar, abordar a paliação, porque, assim, a teoria, a gente aprende uma teoria. O princípio máximo a ser, que você vê na área de saúde, qual é? É salvar vidas! Você vai, todo mundo e fala, eu vou salvar vidas! Você vai salvar vidas! Vou prolongar vidas! E, muitas vezes, não é exatamente prolongar a vida de forma a prolongar. É você dar qualidade de vida dessa pessoa e, muitas vezes, isso é um medo... (M1).

Os profissionais de saúde, ao cuidarem da pessoa em cuidados paliativos, sentem-se impotentes e frustrados em relação à probabilidade de terminalidade da doença, devido à impossibilidade de cura ou à possibilidade real de morte. Não se pode deixar de mencionar que a formação acadêmica prepara o médico e demais profissionais da saúde para salvar vidas ${ }^{(12)}$.

Nessa perspectiva, prestar os cuidados paliativos à pessoa com uma doença crônica requer experiência e conhecimentos específicos, já que os profissionais precisam atender à complexidade das necessidades da pessoa adoecida e de seus familiares de forma integral, promovendo conforto $^{(13)}$.

\section{Ética}

Nesta categoria, os profissionais relataram que um dos maiores dilemas éticos que enfrentavam na oncologia era o sigilo profissional. Isto significava que a pessoa não sabia do seu próprio diagnóstico, na maioria das vezes por questões familiares, e o profissional tinha que manter e respeitar a solicitação de não revelar, mesmo sabendo que, conforme os princípios éticos, é direito da pessoa saber sobre sua doença e diagnóstico. Além disso, foi citada a não reanimação. Se o estado da pessoa fosse grave, com ausência de perspectiva de vida, podiam entrar com uma ordem de não reanimação.

\footnotetext{
A parte de até o paciente saber da própria doença. Então, a gente tem um questionariozinho, que a gente sempre pergunta. Muitas vezes, o próprio médico não fala realmente o que o paciente tem, não porque o paciente não quer saber, mas, às vezes, realmente por quê??... não sei... (E6).
}

Uma das perguntas da anamnese clínica dizia respeito ao conhecimento do paciente sobre o seu diagnóstico. Havia casos em que o paciente não estava informado sobre seu estado de saúde e o profissional não conhecia o motivo e este ter como causa a decisão do médico de não comunicar, como relata E6.

Pessoas com câncer têm direitos assegurados pela legislação brasileira. Tais direitos devem garantir a qualidade de vida e a dignidade de todos. Entretanto, poucas são as pessoas com doenças oncológicas que têm conhecimento dos seus direitos $^{(11,14)}$. Alia-se a isso o fato de que muitos profissionais de saúde, diante da realidade da trajetória do tratamento de pessoas com câncer, 
mesmo sabendo que têm direito de conhecer seu diagnóstico, acabam não informando.

A gente chega um momento que tem família que, por exemplo, tem famílias que não quer que o paciente saiba. A nivel legal, isso, o paciente, ele tem direito, obrigação legal, para a gente informar o paciente. Só que, aí, você tem que ponderar o estado do paciente, de paciente para paciente... (M1).

M1 aponta vivenciar dilema ético diante de uma situação em que a família não quer que a pessoa saiba que tem câncer, como forma de proteção. No entanto, os profissionais sabem que, conforme a Carta dos Direitos dos Usuários dos Serviços de Saúde, art. 3, inciso II, é assegurado à pessoa receber "[...] informações sobre o seu estado de saúde, de maneira clara, objetiva, respeitosa e compreensível quanto a: a) possíveis diagnósticos; b) diagnósticos confirmados;"(15:7-8).

Em relação a esse tema, o Código de Ética dos Profissionais de Enfermagem (CEPE), no art. 42 , cap. II - dos deveres, exorta os profissionais de enfermagem a:

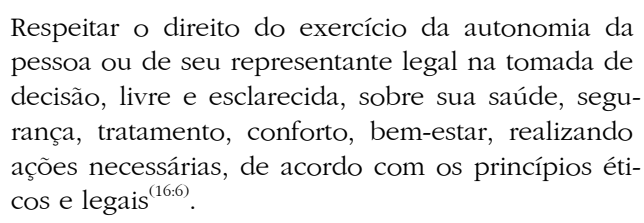
pessoa ou de seu representante legal na tomada de decisão, livre e esclarecida, sobre sua saúde, segurança, tratamento, conforto, bem-estar, realizando ações necessárias, de acordo com os princípios éti$\cos$ e legais ${ }^{(16: 6)}$.

No tocante à comunicação de um prognóstico ruim, não existe uma forma que sirva para todas as pessoas. Cada caso deve ser avaliado, até porque, fornecer informações para quem não deseja ou não está preparado, pode gerar fragilizações ${ }^{(17)}$. Em alguns casos, não querer revelar o diagnóstico de câncer diz respeito à necessidade de o profissional avaliar cada pessoa de forma única e individualizada, e considerar as condições para saber o momento e a forma de revelar o diagnóstico, os cuidados e os tratamentos.

O dilema ético emerge também no momento em que os familiares solicitam que não seja revelado o diagnóstico. E7, por exemplo, tem consciência de que a pessoa com câncer tem o direito de saber sobre o seu diagnóstico, mas a impossibilidade de fazer essa comunicação constitui um dilema ético com o qual precisa lidar.

A gente não deve falar com o paciente, que o paciente não sabe que ele tem câncer, e a gente não deve contar. Então, aí entra um dilema ético [...] a família não quer que a gente conte, mas o paciente, ele tem o direito de saber. (E7).
Muitas vezes, a equipe de saúde encontra-se diante do dilema ético de revelar ou não o diagnóstico para a pessoa com doença oncológica, por não saber se essa será a melhor atitude. Além disso, tal dilema é motivado principalmente pelo desejo dos familiares de prevenir a deterioração da qualidade de vida da pessoa adoecida, nos seus aspectos físicos, emocionais, sociais e ambientais ${ }^{(18)}$.

A ressuscitação do paciente oncológico em estado avançado da doença também pode se constituir em um dilema ético a ser enfrentado pela equipe de saúde. M1, por exemplo, em outra parte do seu relato, aborda a ordem de não ressuscitar. Na sua avaliação como médico, em muitas situações, em consequência do estado avançado da doença, deve entrar com uma ordem de não ressuscitar.

Tem pacientes que, no mesmo quadro dele, a gente reanimaria e tentaria fazer alguma coisa. Tem outros... tem outros que a gente nem tentaria fazer alguma coisa, conversado com a família e tudo. E muitas vezes, a gente pensa que não, mas aí tem uma proteção legal, ai entra essa questão ética. Você vai se proteger legalmente, aí tem outros que a família está ciente, que a família é conversada, que a família diz que não aguenta mais sofrer... (M1).

A ordem médica de não ressuscitar está sempre baseada em conhecimento ético-legal, defendendo a autonomia da pessoa e de seus acompanhantes, e também em alguns critérios pré-definidos, além da discussão com outros profissionais da área da saúde, com o objetivo de proporcionar maior conforto e bem-estar, tanto físico como psíquico e afetivo. Um exemplo é o médico poder optar pela ordem de não ressuscitar, quando faz uma avaliação em determinada pessoa no final da vida, no que se refere à irreversibilidade, e a melhora depende de pesados sacrifícios físicos impostos à pessoa doente ${ }^{(19-20)}$.

Este estudo apresenta como limitações a dificuldade de realizar a coleta de dados, devido à demora de liberação da instituição de saúde, bem como à escassez de estudos relacionados aos dilemas éticos no contexto oncológico. Essas dificuldades reforçam a importância de mais estudos sobre esta temática.

O estudo poderá contribuir para o conhecimento sobre o agir da equipe de saúde no cuidado dirigido às pessoas em tratamento 
oncológico e a seus familiares, além de revelar a importância da tomada de decisão embasada na ética e na moral.

\section{Conclusão}

Os resultados deste estudo mostraram que os profissionais de saúde, em sua maioria, possuíam conhecimento a respeito dos dilemas éticos, definindo-os com clareza e reconhecendo as vivências em que o dilema estava presente. Também foi possível perceber a presença da ambiguidade entre os conceitos de dilema ético e conflito ético.

Os dilemas éticos vivenciados na prática dos profissionais de saúde no cuidado da pessoa em tratamento oncológico estavam relacionados com o cuidado paliativo. Os profissionais questionavam-se quanto à indicação para cuidados paliativos, além de interrogarem-se acerca de até onde podiam intervir no cuidado da pessoa nessa condição; não reanimação, associada à falta de comunicação entre a equipe de saúde e a pessoa a ser cuidada, a fim de respeitá-la e garantir sua autonomia; o desconhecimento quanto a cuidados paliativos, tendo em vista o princípio máximo de salvar vidas; e não revelar o diagnóstico de câncer para a pessoa adoecida, evidenciado pela deficiência de comunicação.

\section{Colaborações:}

1 - concepção, projeto, análise e interpretação dos dados: Maryana Carneiro de Queiroz Ferreira, Marluce Alves Nunes Oliveira e Thamara Arianny Ventin Amorim Oliveira de Assis;

2 - redação do artigo e revisão crítica relevante do conteúdo intelectual: Maryana Carneiro de Queiroz Ferreira, Marluce Alves Nunes Oliveira, Elaine Guedes Fontoura e Manuela Bezerra Pina Oliveira;

3 - aprovação final da versão a ser publicada: Maryana Carneiro de Queiroz Ferreira, Marluce Alves Nunes Oliveira, Keccya dos Santos Nunes Gonçalves e Joselice Almeida Gois.

\section{Referências}

1. Luz KR, Vargas MBO, Schmidtt PH, Barlem ELD, Tomaschewski-Barlem JG, Rosa LM. Problemas éticos vivenciados por enfermeiros oncológicos. Rev Latino-Am Enfermagem. 2015;23(6):1187-94. DOI: 10.1590/0104-1169.0098.2665

2. Rahman MA, Rashid MM, Kernec JL, Philippe B, Barnes SJ, Fioranelli F, et al. A Secure Occupational Therapy Framework for Monitoring Cancer Patients' Quality of Life. Sensors (Basel). 2019;19(23):1-30. DOI: 10.3390/s19235258

3. Kwon JH, Baek SK, Kim BS, Koh SJ, Ahn HK, Lim JH, et al. Surrogate decision making of chemotherapy consent: do we really provide informed consent of chemotherapy for patients? Korean J Intern Med. 2019;34(3):626-33. DOI: $10.3904 / \mathrm{kjim} .2017 .252$

4. Bardin L. Análise de conteúdo. Lisboa (PT): Edições 70; 2016.

5. Marsia S, Khan A, Khan M, Ahmed S, Hayat J, Minhas AMK, et al. Heart transplantation after the circulatory death; The ethical dilema. Indian Heart J. 2018;70(3):442-5. DOI: 10.1016/j. ihj.2018.08.010

6. Panfilis L, Di Leo S, Peruselli C, Ghirotto L, Tanzi S. "I go into crisis when ...": ethics of care and moral dilemmas in palliative care. BMC Palliat Care. 2019;18(70):1-8. DOI: 10.1186/ s12904-019-0453-2

7. Rabadán AT, Tripodoro VA. ¿Cuándo acudir al comité de bioética institucional? El método deliberativo para resolver posibles dilemas. Medicina [Internet]. 2017 [cited 2020 Dec 1];77(6):486-90. Available from: https:// www.medicinabuenosaires.com/indices-de2010-a-2017/volumen-77-ano-2017-no-6-indice/ cuando-acudir-al-comite-de-bioetica-institucionalel-metodo-deliberativo-para-resolver-posiblesdilemas/

8. Oliveira MAN, Rosa DOS. Conflitos e dilemas éticos: vivências de enfermeiras no centro cirúrgico. Rev baiana enferm. 2016;30(1):344-55. DOI: $10.18471 /$ rbe.v1i1.14237

9. Lima CP, Machado MA. Cuidadores principais ante a experiência da morte: seus sentidos e significados. Psicol ciênc prof. 2018;38(1):88-111. DOI: 10.1590/1982-3703002642015 
10. Baère TD, Faustino AM, Miranda AF. A importância da prática interdisciplinar da equipe de saúde nos cuidados paliativos. Rev Portal Divulgação [Internet]. 2017 [cited 2020 Oct 5];53:5-19. Available from: https://revistalongeviver.com.br/index.php/ revistaportal/article/view/673

11. Wouters S, Exel JV, Baker R, Brouwer WBF. Priority to End of Life Treatments? Views of the Public in the Netherlands. Value Health. 2017;20(1):107-17. DOI: 10.1016/j.jval.2016.09.544

12. Ribeiro JR, Poles K. Cuidados Paliativos: Prática dos Médicos da Estratégia Saúde da Família. Rev bras educ méd. 2019;43(3):62-72. DOI: $10.1590 / 1981-52712015 \mathrm{v} 43 \mathrm{n} 3 \mathrm{rb} 20180172$

13. Sekse RJT, Hunskår I, Ellingsen S. The nurse's role in palliative care: A qualitative meta-synthesis. J Clin Nurs. 2018;27(1-2):e21-e38. DOI: 10.1111/ jocn. 13912

14. Agarwal R, Saltz LB. Understanding the Right to Try Act. Clin Cancer Res. 2020;26(2):340-3. DOI: 10.1158/1078-0432.CCR-19-2015

15. Brasil. Ministério da Saúde. Carta dos direitos dos usuários da saúde [Internet]. 3a ed. Brasília (DF); 2011 [cited 2019 Nov 20]. Available from: http:// www.conselho.saude.gov.br/biblioteca/livros/AF_ Carta_Usuarios_Saude_site.pdf

16. Conselho Federal de Enfermagem. Resolução Cofen no 564, de 6 de novembro de 2017. Aprova o novo Código de Ética dos Profissionais de Enfermagem [Internet]. Brasília (DF); 2017 [cited 2020 Oct 22]. Available from: http://www.cofen. gov.br/resolucao-cofen-no-5642017_59145.html

17. Moritz RD. Cuidados paliativos no final da vida [Internet]. Florianópolis: CRM-SC; 2016 [cited 2019 Nov 15]. Available from: http://arquivos.cremesc. org.br/publicacao/Cuidados_Paliativos_Livro.pdf

18. Victor A, George CE, Inbaraj LR, Norman G. Benefit or Harm? A Study on Impact of Collusion on the Quality of Life among Palliative Care Patients. Indian J Palliat Care. 2018;24(1):61-6. DOI: 10.4103/IJPC.IJPC_111_17

19. Paixão QL, Oliveira MAN, Fontoura EG, Freitas KS. Dilemas éticos no fazer/agir do enfermeiro diante da parada cardiorrespiratória em terapia intensiva. Rev baiana enferm. 2019;33:e27920. DOI: 10.18471/rbe.v33.27920

20. Eidt V, Bruneri GD, Bonamingo EL. Ordem de não reanimar sob a perspectiva de pacientes oncológicos e seus familiares. Mundo saúde. 2017;41(3):395-403. DOI: 10.15343/0104-7809.20 174103395403

Recebido: 4 de fevereiro de 2021

Aprovado: 13 de maio de 2021

Publicado: 8 de junho de 2021

A Revista Baiana de Enfermagem utiliza a Licença Creative Commons - Atribuição-NãoComercial 4.0 Internacional. https://creativecommons.org/licenses/by-nc/4.0/

Este artigo é de acesso aberto distribuído sob os termos da Licença Creative Commons (CC BY-NC). Esta licença permite que outros remixem, adaptem e criem a partir do seu trabalho para fins não comerciais. Embora os novos trabalhos tenham de lhe atribuir o devido crédito e não possam ser usados para fins comerciais, os usuários não têm de licenciar esses trabalhos derivados sob os mesmos termos. 